\title{
Paclitaxel-loaded trimethyl chitosan-based polymeric nanoparticle for the effective treatment of gastroenteric tumors
}

\author{
RONG-FENG SONG ${ }^{1}$, XIAO-JUN LI ${ }^{2}$, XIAO-LIANG CHENG ${ }^{3}$, AI-RONG FU ${ }^{4}$, \\ YAN-HUA WANG ${ }^{1}$, YAN-JUN FENG ${ }^{1}$ and YAN XIONG ${ }^{1}$ \\ Departments of ${ }^{1}$ Gastroenterology, ${ }^{2}$ Hepatic Neoplasms Surgery, ${ }^{3}$ Thoracic Surgery and ${ }^{4}$ Pathology, \\ Cancer Hospital of Jiangxi Province, Nanchang, Jiangxi 330029, P.R. China
}

Received March 22, 2014; Accepted June 25, 2014

DOI: $10.3892 / o r .2014 .3344$

\begin{abstract}
Gastroenteric cancer is one of the most prevalent cancers and is responsible for most cancer-related deaths worldwide. Paclitaxel (PTX), a classical microtubule inhibitor, is indicated in the treatment of gastric/gastroenteric cancers. In the present study, trimethyl chitosan (TMC)-loaded PTX (TMC-PTX) was prepared and evaluated for its therapeutic effect in gastric cancers. A spherical shaped nanosized TMC-PTX particle was formed with high loading capacity $(\sim 30 \%)$ for PTX. The nanoparticles (NPs) showed a sustained release pattern $(\sim 70 \%)$ for up to $96 \mathrm{~h}$ of study period. The positively charged NPs were preferentially internalized by Caco-2 cells. TMC-PTX inhibited the gastric cell proliferation with an $\mathrm{IC}_{50}$ value of $0.6 \mu \mathrm{g}$ in NCI-N87 cells while it was $1.26 \mu \mathrm{g}$ in the SGC-7901 cell line after $24 \mathrm{~h}$ exposure. The apoptosis assay (Annexin V/PI) showed a large presence of cells in the early and late apoptosis chamber, while cell cycle analysis showed a marked G2/M phase arrest (50-60\%) in NCI-N87 and SGC-7901 cell lines indicating its potent anti-proliferative effect. The in vivo antitumor study in NCI-N87 and SGC-7901 bearing xenograft model showed a superior chemotherapeutic efficacy for TMC-PTX NP. The NP group significantly reduced the tumor growth with no obvious sign of systemic side-effects (safety). Collectively, our results suggest that the microtubule inhibitory effect of PTX-loaded polymer NP could be a promising system for the treatment of gastroenteric cancers.
\end{abstract}

\section{Introduction}

Gastroenteric cancer (GE) is one of the most common cancers in the world with high mortality rates worldwide (1). Although the incidence of GE tumor has decreased considerably, it

Correspondence to: Dr Rong-Feng Song, Department of Gastroenterology, Cancer Hospital of Jiangxi Province, Nanchang, Jiangxi 330029, P.R. China

E-mail: song100973@gmail.com

Key words: paclitaxel, trimethyl chitosan, gastroenteric cancer, nanoparticles, anti-proliferative effects remains the second leading cause of cancer-related mortality. The current treatment strategy includes surgical removal followed by adjuvant chemotherapy $(2,3)$. Therefore, reoccurrence and relapse highly depend on the efficiency of the chemotherapeutic treatment. In this regard, paclitaxel (PTX), a microtubule destabilizing agent, has shown considerable potential against gastric-related cancers (4). PTX exerts its effect by facilitating tubulin polymerization and by stabilization of microtubules in G2-M phase arrest and mitotic cell death (5). However, its poor aqueous solubility and low therapeutic index limits its clinical application (6). To counter this problem, Taxol, a cremophore based alcoholic mixture, was introduced and although it improved the solubility, it was associated with severe adverse effects including neurotoxicity and nephrotoxicity (7). Consequently, cremophore free albuminbound PTX nanoparticles (NPs; Abraxane) were launched to overcome the side-effects of Taxol. Although Abraxane was effective, it showed a poor colloidal stability in vivo (8). Therefore, the need for biocompatible, stable, tunable release controlled delivery system for PTX remains.

$\mathrm{N}, \mathrm{N}, \mathrm{N}$-trimethyl chitosan (TMC) is a water soluble cationic polyelectrolyte useful for the oral and intravenous drug delivery of PTX (9). TMC is a biocompatible and biodegradable polymer which can be effectively used to form NPs of $150-200 \mathrm{~nm}$ via ionic gelation process with tripolyphosphate (TPP) as an anionic counterpart (10). Previously, many therapeutic moieties including antioxidants, enzymes, vaccines, antimicrobials and small molecules were successfully encapsulated and administered in vivo (11). The encapsulation of PTX in TMC NPs could effectively overcome the solubility issues, provide a controlled release profile and can prolong the half-life of drug in the in vivo conditions. Moreover, the excellent mucoadhesive property of TMC would further facilitate the PTX transport across the GE cancer cells in the body (12).

Thus, the purpose of the present study was to prepare PTX-encapsulated TMC NPs and to investigate their effect on the gastroenteric tumors. To achieve this purpose, nanosized TMC NPs of $200 \mathrm{~nm}$ were prepared by cross-linking with TPP counter ion. Various physicochemical characteristics including particle size, surface charge, loading efficiency, release kinetics and stability were investigated. Biological investigations including cellular uptake and cytotoxicity assays were performed. Most importantly, antitumor efficacy was carried 
out in tumor bearing xenograft nude mice to establish its tumor regression and safety profile.

\section{Materials and methods}

Materials. PTX, chitosan (viscosity 20-200 cps; degree of acetylation $85 \%$ ) and sodium tripolyphosphate (TPP) were procured from Sigma-Aldrich (Shanghai, China) and used as delivered. Dimethyl sulfate was purchased from Vetec (Brazil). All other chemicals were of analytical grade and used without further modifications.

\section{Methods}

Synthesis of TMC. The N-TMC polymer was synthesized as previously reported. Briefly, methylation of chitosan was carried out with dimethyl sulfate at $70^{\circ} \mathrm{C}$. Approximately $1 \mathrm{~g}$ of chitosan was reacted with $16 \mathrm{ml}$ of dimethyl sulfate and the rest of the methylation process was followed as mentioned in the literature (13).

Preparation of PTX-loaded TMC NPs. PTX-TMC NPs were formulated by ionic gelation method using complexation between oppositely charged macromolecules (14). Briefly, PTX was dissolved in a hydro-alcoholic TMC solution and gently vortexed for $30 \mathrm{~min}$. TPP at a concentration ranging from 10 to $50 \% \mathrm{w} / \mathrm{w}$ was added dropwise on the TMC solution. The mixture was sonicated for $20 \mathrm{~min}$ followed by dynamic light scattering experiments.

Particle size and $\zeta$-potential measurements. Zetasizer (Malvern, UK) was used to measure the hydrodynamic particle size, polydispersity index and $\zeta$-potential measurements using dynamic light scattering (DLS) techniques. All measurements were performed at a fixed angle of $90^{\circ}$ at $25^{\circ} \mathrm{C}$ room temperature. The results were expressed as the size \pm SD.

Morphology. The structural morphology was examined by transmission electron microscopy (TEM). Briefly, liquid sample was placed in a carbon-coated copper grid and counter stained with phosphotungstic acid, followed by air drying for $2 \mathrm{~h}$. The surface topography was further confirmed by the atomic force microscopy (AFM) where in samples were instilled on the MICA surface and air dried for $2 \mathrm{~h}$.

PTX loading efficiency. Loading efficiency was calculated from the total amount of drug added vs. amount of drug entrapped in the NPs. Briefly, PTX-TMC NP was filtered by Amicon centrifugal filter by centrifuging at a high speed of 5,000 rpm for $10 \mathrm{~min}$. The filtrate was analyzed for unentrapped drug by the HPLC method. The mobile phase (acetonitrile;methanol;water, 28;25;47, pH 3.2) was set at $1 \mathrm{ml} / \mathrm{min}$ with an absorbance of $250 \mathrm{~nm}$.

In vitro release study. PTX-TMC NP was centrifuged at $10,000 \mathrm{x} g$ for $5 \mathrm{~min}$ to separate the free PTX from the conjugated one. The supernatant was removed and PTX-TMC was collected and re-suspended in required amount of distilled water. The samples were placed in a dialysis bag which was in turn placed in release media containing conical tube in a shaker bath $\left(37^{\circ} \mathrm{C}\right)$. At a specified time, $1 \mathrm{ml}$ of release media (phosphate-buffered saline) was removed and replaced with equal amount of fresh buffer. The amount of drug released was plotted against time.

In vitro cellular uptake. The cellular uptake of PTX-TMC NP was analyzed by the HPLC method. Briefly, $1 \times 10^{6} \mathrm{Caco}-2$ cells were seeded into 6-well plates and allowed to attach for $18 \mathrm{~h}$. The cells were treated with free PTX and PTX-TMC NP and incubated for $6 \mathrm{~h}$. The cells were washed, trypsinized and lysed by high sonication. The resultant solutions were centrifuged and supernatant was used to quantify the amount of drug internalized.

Cytotoxicity assay. The gastric cancer cell lines NCI-N87 and SGC-7901 were seeded into 96-well plates at a density of $1 \times 10^{6}$ cells/well in RPMI media supplemented with $10 \%$ FBS and incubated at $37^{\circ} \mathrm{C}$ for $24 \mathrm{~h}$. Cells were then exposed to various doses of blank, free PTX and PTX-TMC and further incubated for $24 \mathrm{~h}$. The cells were washed and treated with $25 \mu \mathrm{l}$ of MTT $(5 \mathrm{mg} / \mathrm{ml})$ and kept in the dark for $4 \mathrm{~h}$. The formazan crystals were extracted and absorption was noted at $570 \mathrm{~nm}$ using an ELISA (ELX800 Bio-Tek, Winooski, VT, USA) plate reader.

Apoptosis study. The apoptosis of gastric cells was investigated by flow cytometry (BD FACS Atira II, BD Company, USA) with a cell apoptosis kit (Alexa Fluor 488 Annexin V/Dead cell apoptosis kit with Alexa Fluor 488 Annexin V and PI for flow cytometry; Invitrogen). NCI-N87 and SGC-7901 cells were seeded in 6-well plates at a density of $3 \times 10^{5}$ cells/well with RPMI media for $24 \mathrm{~h}$. Cells were then exposed to various doses of free PTX and PTX-TMC and further incubated for $24 \mathrm{~h}$. The cells were extracted with trypsin and PI and fluorescence labeled Annexin V was added as per the manufacturer's instructions. The apoptotic cell ratio was calculated by the ratio of apoptosis cells and dead cells to total cells.

Cell cycle analysis. NCI-N87 and SGC-7901 cells were seeded in 6-well plates at a density of $3 \times 10^{5}$ cells/well with RPMI media for $24 \mathrm{~h}$. Cells were exposed to various doses of free PTX and PTX-TMC and further incubated for $24 \mathrm{~h}$. The cells were washed and incubated with propidium iodide (PI; $20 \mu \mathrm{g} / \mathrm{ml}$ ) for $30 \mathrm{~min}$. The DNA content was measured for 10,000 events for each sample by flow cytometry (BD FACS Atira II, BD Company) and the data was plotted using Cell Quest software.

In vivo antitumor efficacy. All animal experiments were carried out according to the guidelines set by the Animal Ethics Committee (AEC), Cancer Hospital of Jiangxi Province, China. The animals were provided good care throughout the study period under $12 \mathrm{~h}$ day/night cycle. Female SCID nude mice (7 weeks) were used to grow tumor subcutaneously. Gastric cancer NCI-N87 cells $\left(5 \times 10^{6}\right)$ were subcutaneously injected into the left flank of each mouse. The tumor growth was monitored until it reached an average tumor size of $100-150 \mathrm{~mm}^{3}$. The mice were randomly divided into 4 groups with 8 mice in each group. Each group was administered blank vehicle, free PTX and PTX-TMC ( $5 \mathrm{mg} / \mathrm{kg}$ PTX equivalent) 2 times a week for 14 days with one group being untreated control. The tumor 

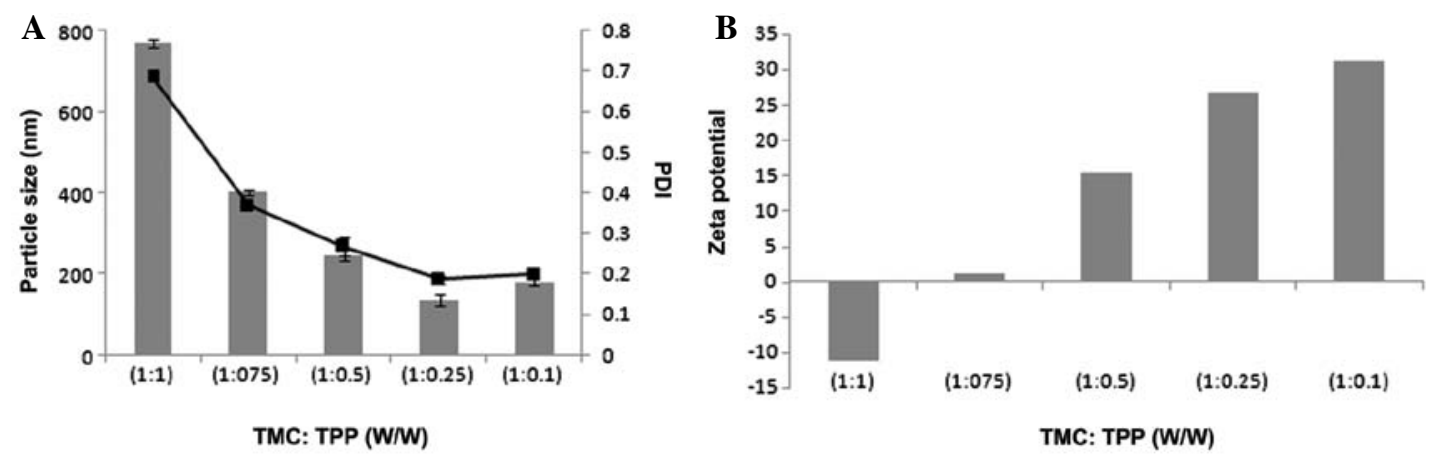

C

D
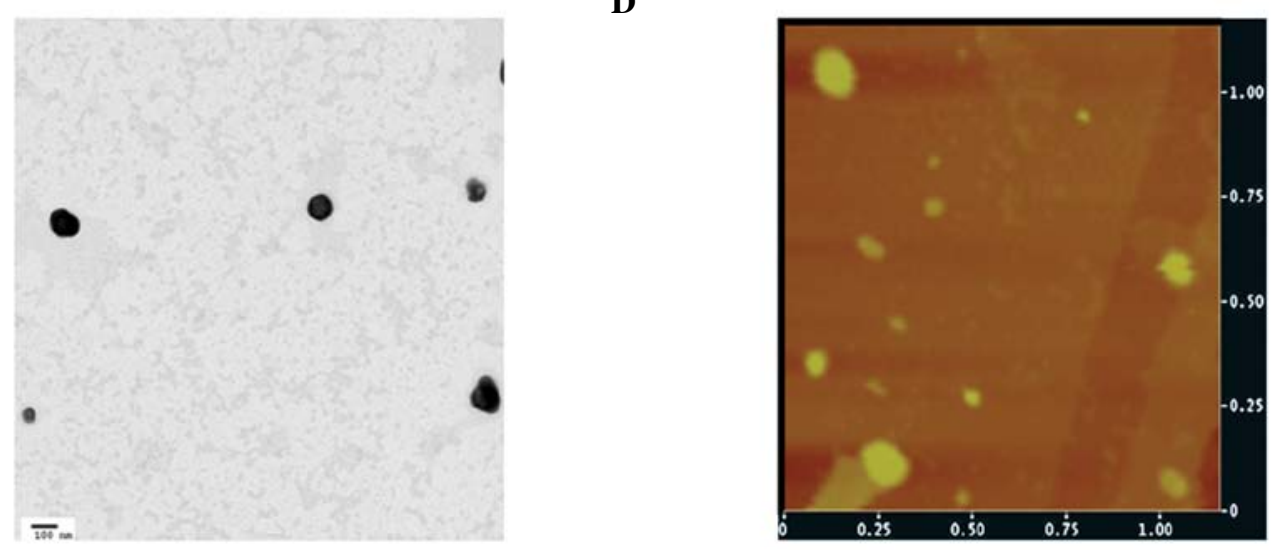

Figure 1. Physicochemical characterization of TMC-PTX nanoparticles. Effect of different weight ratios of TMC:TPP (A) particle size and PDI, and (B) $\zeta$-potential. (C) TEM images of TMC-PTX, (D) AFM images. TMC-PTX, trimethyl chitosan-loaded paclitaxel; TPP, tripolyphosphate; TEM, transmission electron microscopy; AFM, atomic force microscopy.

size was measured twice weekly via caliper and tumor volume (V) was calculated by using the formula: $\mathrm{V}=1 / 2\left[\mathrm{~L} \times(\mathrm{W})^{2}\right]$, $\mathrm{L}=$ length and $\mathrm{W}=$ width.

\section{Results and Discussion}

Preparation of TMC-TPP NPS. The ionic gelation method is considered one of the most suitable methods for the preparation of NPs. In this method, charged macromolecules electrostatically interact with the oppositely charged species resulting in the formation of stable carrier. Such physical cross-linking process eliminates the undesirable side-effects of chemical cross-linkers (15). In this study, various weight ratios of TPP were complexed with positively charged TMC moiety to observe a nanosized particle with uniform size distribution. Fig. 1A shows the influence of TPP ratio on the final size of blank NP. The particle size was big when equal proportion of both complexing moieties was added, while the particle size decreased gradually when the TPP concentration decreased. In particular, at 1:0.25 (TMC-TPP) ratios it undergoes ionic gelation and precipitation to yield a nanosize particle with uniform size distribution (PDI $\sim 0.15$ ).

Generally, specific ratio of TMC and TPP plays an important role in the NP structure which is largely governed by the neutralization process. The TPP containing $\mathrm{P}_{3} \mathrm{O}_{10}{ }^{5}$ - ions neutralizes the chitosan $-\mathrm{NH}_{3}{ }^{+}$amino groups (16). In the case of TMC, this process becomes slightly complex due to the presence of multiple functional groups including quaternary site $\left(-\mathrm{N}\left(\mathrm{CH}_{3}\right)_{3}{ }^{+}\right)$, monomethylated $\left(-\mathrm{N}\left(\mathrm{CH}_{3}\right)_{2} \mathrm{H}^{+}\right)$, dimethyl- ated $\left(-\mathrm{N}\left(\mathrm{CH}_{3}\right) \mathrm{H}_{2}{ }^{+}\right)$and even pure protonated amine site $\left(-\mathrm{NH}_{3}^{+}\right)(17)$.

Similarly, $\zeta$-potential is an important indicator of NP formation. At a higher concentration of TPP, surface charge was slightly negative due to the high cross-linking density of anionically charged TPP (Fig. 1B). The $\xi$-potential, however, reversed to strong positive charge when the concentration of negatively charged species decreased. At this stage, cationic surface charge of NPs attributed to the large presence of cationic head groups of chitosan (18). According to the literature, physical stability of NPs is good when the $\zeta$-potential value is $>20 \mathrm{mV}$. Therefore, TMC:TPP (1:0.25) ratio which produced nanosized particle with strong positive charge was selected to incorporate PTX.

Preparation of TMC-PTX NPs. Table I presents the effect of PTX incorporation on the physicochemical property of TMC-TPP NPs. The particle size of TMC-PTX NP increased more than $\sim 25 \mathrm{~nm}$ for the addition of PTX indicating a successful entrapment within the NP. This finding is in agreement with previously published reports which showed that based on the molecular weight and charge of small molecules, size varied between $50-200 \mathrm{~nm}(17,19)$. In the present study, PTX possessed a small molecular weight and a neutral charge which does not interfere in the electrostatic interaction process between TMC and TPP. It was also shown that PTX was efficiently incorporated into the NP matrix with $95 \%$ of entrapment efficiency. Similarly, loading capacity was also found to be more than $25 \%$ of total NP mass. 
Table I. Physicochemical characterization of TMC-PTX nanoparticles (NPS).

\begin{tabular}{lccccc}
\hline & $\begin{array}{c}\text { Size } \\
(\mathrm{nm})\end{array}$ & PDI & $\begin{array}{c}\text { S-potential } \\
(\mathrm{mV})\end{array}$ & $\begin{array}{c}\text { Entrapment } \\
\text { efficiency }(\%)\end{array}$ \\
\hline Blank TMC NP & $135.4 \pm 1.75$ & $0.168 \pm 0.018$ & $26.65 \pm 0.98$ & - & $\begin{array}{c}\text { Loading } \\
\text { efficiency }(\%)\end{array}$ \\
TMC-PTX NP & $158.8 \pm 1.4$ & $0.211 \pm 0.002$ & $25.82 \pm 1.51$ & $95.8 \pm 2.3$ & $27.8 \pm 1.98$ \\
\hline
\end{tabular}

TMC-PTX, trimethyl chitosan-loaded paclitaxel.

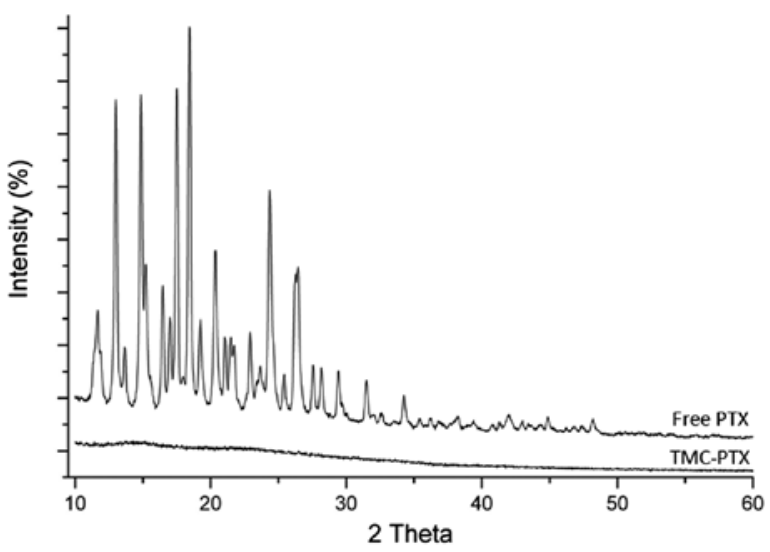

Figure 2. XRD diffraction patterns of free PTX and TMC-PTX nanoparticles. TMC-PTX, trimethyl chitosan-loaded paclitaxel.

Morphology and topography. TEM was used to confirm the morphology and structure of TMC-PTX NP. The imaging showed spherical shaped particles with perfect boundary with individual objects. The particles were uniformly distributed in the copper grid. A dense black core might be due to the high electron density of phosphate group of TPP complex (Fig. 1C). The TEM size $(\sim 100 \mathrm{~nm})$ was consistent with the hydrodynamic particle size measured by Zetasizer. The shape was further confirmed by AFM analysis (Fig. 1D). The NPs were uniformly spread and flatted on the cover slip with clear round and circular shape. The particle angles were not as sharp as observed under TEM as the analysis was performed in contact mode that may distort the actual structure of particles.

XRD analysis. XRD analysis was performed to confirm the nature of drug incorporation. XRD patterns of free PTX and TMC-PTX are presented in Fig. 2. Free PTX showed numerous sharp characteristic peaks of $2 \Theta$ between $10-30^{\circ}$ indicating its prominent crystalline nature. TMC-PTX pattern, however, did not show any such peaks suggesting the presence of drug in the amorphous form in the NPs.

In vitro release study. The in vitro release profiles of TMC-PTX NP are presented in Fig. 3. The NPs exhibited a biphasic release profile with $>25 \%$ of drug released within $12 \mathrm{~h}$ of study period, followed by a relatively sustained release pattern up to $96 \mathrm{~h}(\sim 75 \% \mathrm{PTX})$. The initial fast release attributed to the dissolution and diffusion of PTX either located on the surface or poorly entrapped in the polymer matrix. The sustained release however enabled the slow diffusion of drug from the

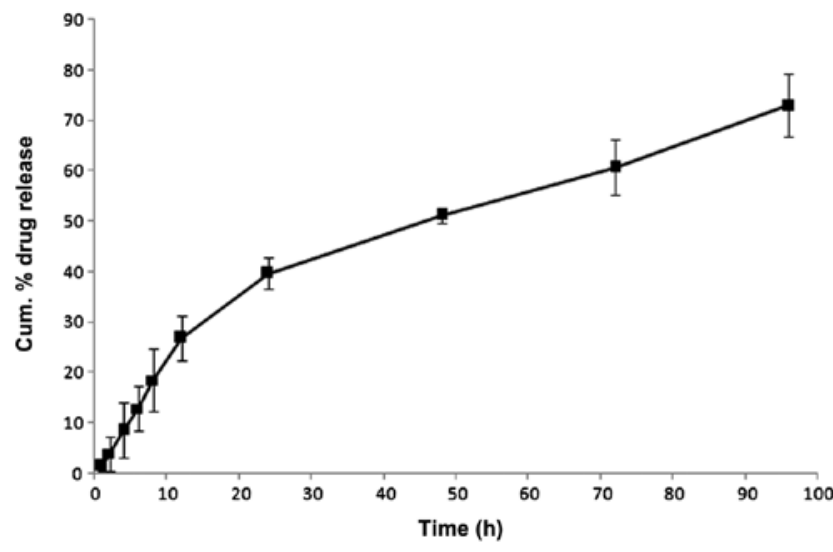

Figure 3. In vitro release profile of PTX from TMC-PTX nanoparticles in physiological $\mathrm{pH} 7.4$ at $37^{\circ} \mathrm{C}$. TMC-PTX, trimethyl chitosan-loaded paclitaxel.

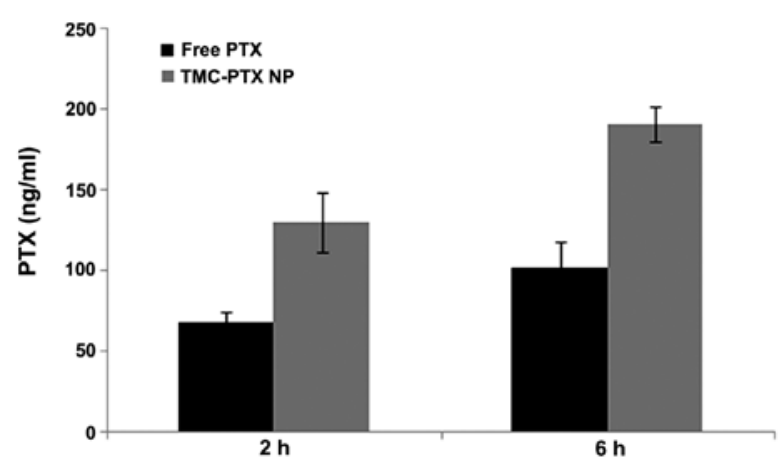

Figure 4. Cellular internalization of free PTX and TMC-PTX in Caco-2 cells incubated for 2 and $6 \mathrm{~h}$ respectively. TMC-PTX, trimethyl chitosan-loaded paclitaxel.

hydrophobic core matrix. Specifically, hydrophobic interaction between PTX and methylated chitosan could be anticipated. PTX molecule has a hydrophobic region and a partially hydrophilic region with hydroxyl and secondary amine groups which can form hydrogen bonds with chitosan molecules. Such slow release has significant importance in the systemic application wherein the drug will be available for therapeutic action in a steady manner. Furthermore, release profile best fitted the Higuchi model $\left(r^{2}=0.9865\right)$ suggesting a diffusion controlled release mechanism. The Korsmeyer-Peppas model was applied to gain further insight into the mechanisms of release. An $n$ value of 0.65 indicates the presence of anomalous transport which means a combination of drug diffusion and polymer matrix relaxation. 
A

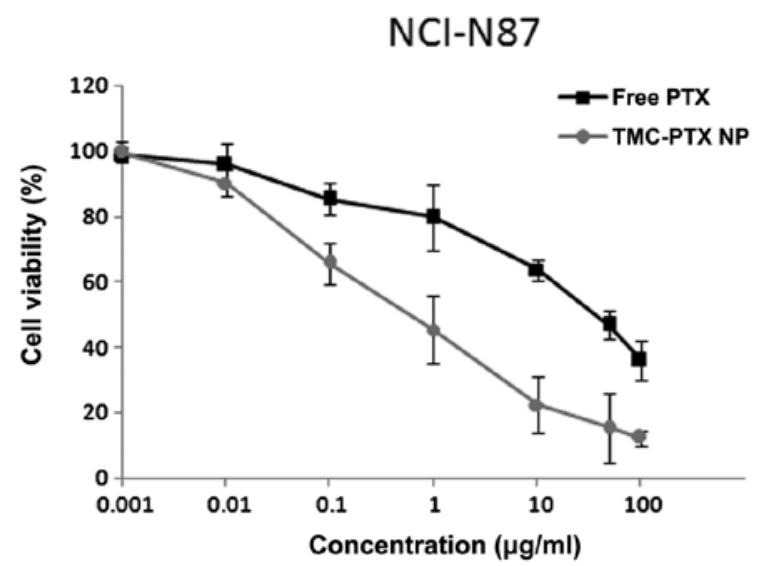

B

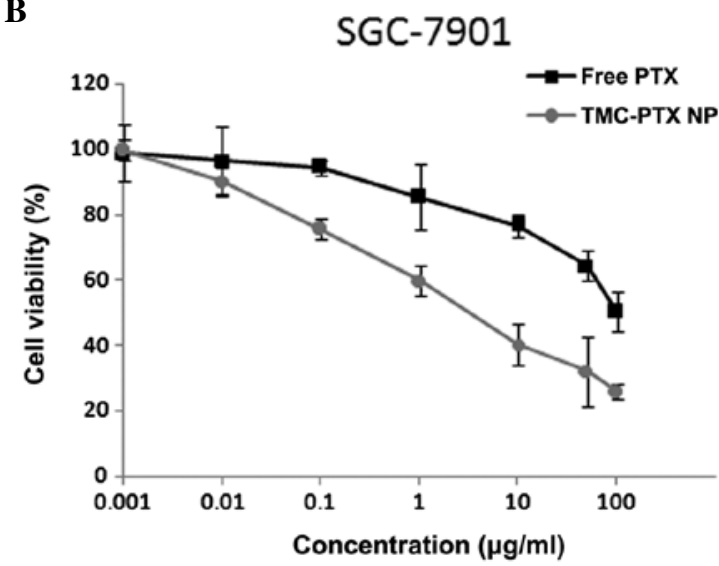

Figure 5. In vitro cytotoxicity profile of free PTX and TMC-PTX against (A) NCI-N87 and (B) SGC-7901 cells after 24 h exposure. Data are expressed as mean $\pm \mathrm{SD}(\mathrm{n}=8)$. TMC-PTX, trimethyl chitosan-loaded paclitaxel.

Cellular uptake. Intracellular drug uptake studies were performed using HPLC. Since gastroenteric cancer lies in the gastrointestinal tract, cellular uptake of free PTX and TMC-PTX was studied in Caco-2 cells. The drug internalization in Caco-2 cells from both groups are presented in Fig. 4. After $2 \mathrm{~h}$ of incubation, a considerable amount of PTX was accumulated in cells with significantly $(\mathrm{P}<0.005)$ higher internalization of PTX from NPs over free drug. As expected, the same trend was observed at a longer incubation time $(6 \mathrm{~h})$ with nearly 2-fold higher uptake from both groups. Higher drug accumulation from TMC-PTX might be attributed to the positive charge of NPs that facilitated the drug internalization. Generally, anionic heparin covers the outer cell surface that may be responsible for the binding affinity/favorable interaction with cationic species (20). Furthermore, it has been reported that cellular uptake is size-dependent and smaller sized NPs were preferably uptaken over larger or bulk sized NPs (21). Collectively, it can be said that positive charge and nanosize of particles contributed to the enhanced cellular uptake of TMC-PTX.

Cytotoxicity assay. Advanced gastroenteric tumors are life threatening diseases that present formidable challenges. Many double and triple drug combination regimens have been shown to evoke poor clinical response and are often associated with severe side-effects. Therefore, the present study focused on the NP-based PTX delivery to overcome the drawbacks associated with single drug or multiple drug combinations. Cytotoxicity assays were performed on gastric cancer cell lines (NCI-N87 and SGC-7901) to confirm the superior effect of TMC-PTX. Free PTX as well as TMC-PTX inhibited gastric cancer cell proliferation in a dose-dependent manner (Fig. 5A and B). Specifically, TMC-PTX exhibited the maximum anti-proliferative effects by comparison to free PTX, consistent with the cellular uptake observations which showed higher intracellular accumulation for the NPs. NCI-N87 was relatively more sensitive to PTX than SGC-7901 which was slightly less responsive to the drug. The $\mathrm{IC}_{50}$ value of free PTX and TMC-PTX in NCI-N87 cell was 0.6 and $12.5 \mu \mathrm{g}$, respectively. In the case of SGC-7901 cells, IC $_{50}$ values were significantly higher at 1.26 and $28.8 \mu \mathrm{g}$, respectively for both groups. The difference in cytotoxic effect on cell lines may be attributed to the difference in genetic origin and indigenous biological behavior. It can be expected that at longer incubation periods, many cell populations enter the $\mathrm{G} 2$ and $\mathrm{M}$ phases at which it is highly active. Drug-free blank polymeric NPs, however, did not exhibit obvious cytotoxicity in either cell line in all tested concentrations indicating high biocompatibility.

Apoptosis study. PTX binds to the $\beta$-subunit of the $\alpha / \beta$-tubulin dimerin in the microtubule that induces the polymerization of tubulin which in turn inhibits the growth of rapidly dividing cells resulting in apoptosis (22). To confirm the cytotoxic potential of free PTX and TMC-PTX, cells were treated with Annexin V and PI and analyzed using flow cytometry. Annexin V (35-36kDa), a Ca ${ }^{2+}$-dependent phospholipid-binding protein shows high affinity for phospholipid phosphatidylserine lining the outer plasma membrane in cell apoptosis (23). PI is a standard molecular probe used to distinguish viable cells from nonviable cells. Generally, viable cells having intact membranes do not interact with PI, while membranes of dead cells are permeable to PI (24). Therefore, Annexin V-FITC/ PI staining can distinguish early apoptosis from late apoptosis in cell populations. As expected, a considerable proportion of cells was in early and late apoptosis stages suggesting the potent action of free drug and drug-loaded NP (Fig. 6A and B). Specifically, TMC-PTX NP showed 25 and $40 \%$ cell death in early and late apoptosis which was significantly higher than the free drug (15 and $28 \%$, respectively) in NCI-N87 cells. The trend was similar in SGC-7901 cells; however, percentage of cell populations in both stages was relatively lower compared with NCI-N87 cells. The apoptosis result was consistent with the higher cytotoxicity potential of nanoparticulate formulations in these cell lines (25). These observations indicate that PTX was intact in the TMC NP and released in a controlled manner to induce apoptosis.

Cell cycle analysis. The toxicity of PTX and TMC-PTX was further confirmed by cell cycle analysis. It is known that PTX induced cell cycle arrest by impairing mitosis and PTX-induced $\mathrm{G} 2 / \mathrm{M}$ arrest is associated with the breakage of double-strain DNA and considerable chromosome damage (26). In this 
A

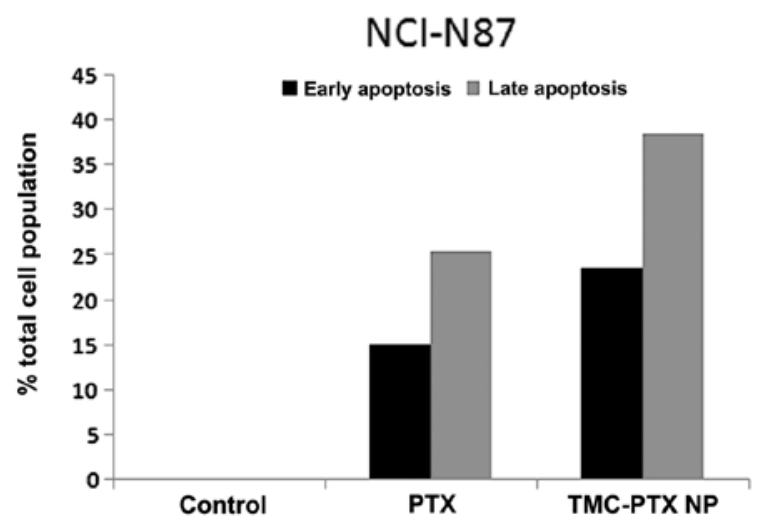

B

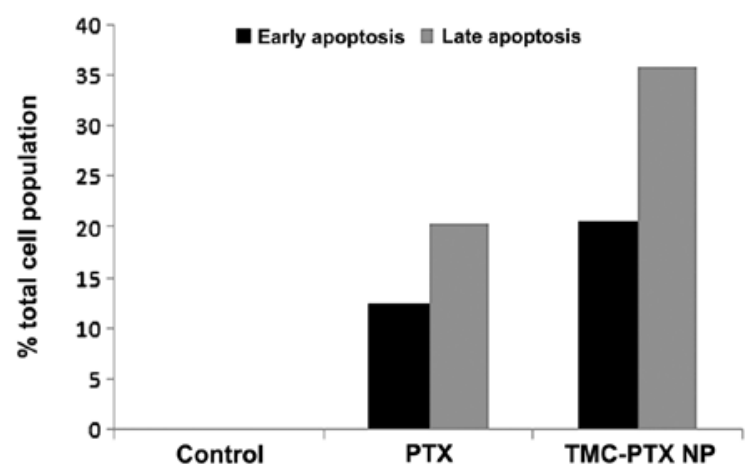

Figure 6. Annexin V/PI based apoptosis assay on (A) NCI-N87 and (B) SGC-7901 cells upon exposure to respective formulations. TMC-PTX, trimethyl chitosan-loaded paclitaxel.

A

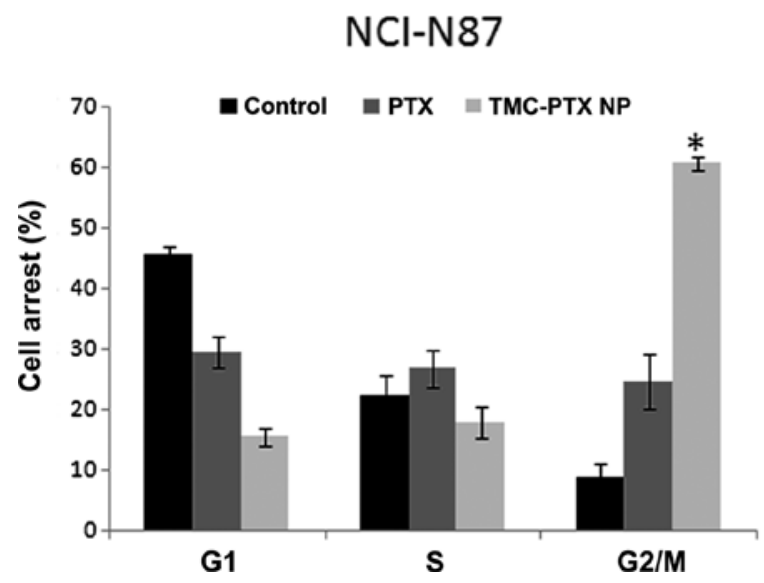

B

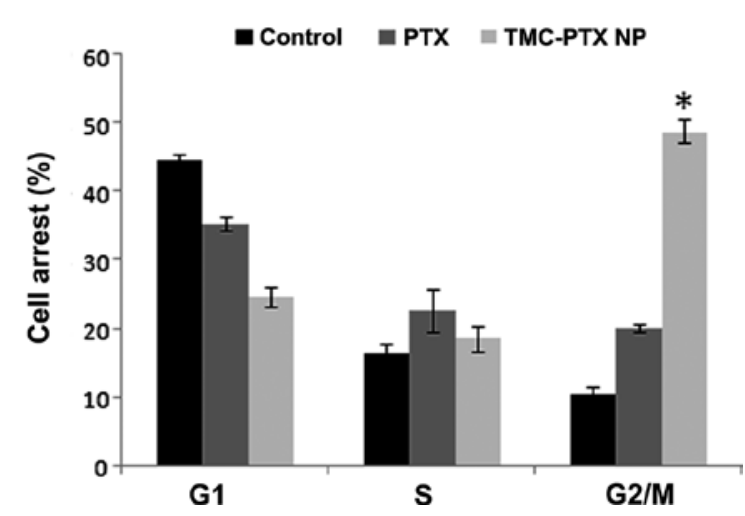

Figure 7. Cell cycle analysis of (A) NCI-N87 and (B) SGC-7901 cells after treatment with free PTX and TMC-PTX nanoparticles. TMC-PTX, trimethyl chitosan-loaded paclitaxel.

perspective, it follows that increased $\mathrm{G} 2 / \mathrm{M}$ phase arrest is associated with the inhibition of cell division and cell growth arrest (27). Results of the present study clearly showed the presence of cell populations in different phases upon treatment with respective formulations. As can be seen, nearly $45 \%$ of cells were present in the G1 phase while only $<10 \%$ of cells were in the G2/M phase in the control/untreated cells in NCI-N87 cells. Upon PTX treatment, however, G1 phase population decreased to $28 \%$ with nearly $25 \%$ of cells having entered the $\mathrm{G} 2 / \mathrm{M}$ where PTX exhibited the maximum action (Fig. 7A and B). Notably, TMC-PTX exhibited a marked $60 \%$ of cells in G2/M phase which is almost 2-fold higher than compared to the free PTX group and 6-fold higher than the control. A considerable proportion of cells was present in the subG0 (11\%) phase of the cell cycle, while $50 \%$ of cells were present in the G2/M phase in the case of SGC-7901 cells. These observations clearly reveal the fact that PTX exhibits prominent $\mathrm{G} 2 / \mathrm{M}$ phase cell accumulation. These results are consistent with the above cytotoxicity study and apoptotic assay.

Antitumor efficacy. The antitumor efficacy study was investigated on NCI-N87 and SGC-7901 gastric cancer cells bearing xenograft tumor models. As can be seen, the tumor in the untreated group grew rapidly and attained maximum size at the study period. In contrast, free PTX and TMC-PTX significantly inhibited the tumor growth in both xenograft mice models. The tumor volume was the same for the two groups until day 7, after which time the tumor growth was accelerated in the PTX group and the tumor size was significantly $(\mathrm{P}<0.005)$ different from each other at the end of day 20. In a key finding, in the case of TMC-PTX, the tumor volume gradually increased until day 10 after which complete tumor regression was observed and tumor size did not increase further in either tumor model (shrunk) (Fig. 8A and B). It has previously been reported that repeated administrations of PTX-NPs could markedly increase the drug in tumor targets, resulting in maximum therapeutic efficacy (28). The free PTX group, however, showed constant increment in the tumor volume throughout the study period although it was less than that of control. Specifically, TMC-PTX showed 70 and $64 \%$ tumor regression in NCI-N87 and SGC-7901 xenograft mice, compared to only 30 and $25 \%$ growth inhibition, respectively, in the case of free PTX. The result was consistent with our in vitro cell proliferations and apoptosis observations which were prominent for TMC-PTX.

Tumor weight is an indicator of drug-related systemic toxicity and therefore tumor weights were recorded simultaneously with the tumor volume measurements. From the results, 
A

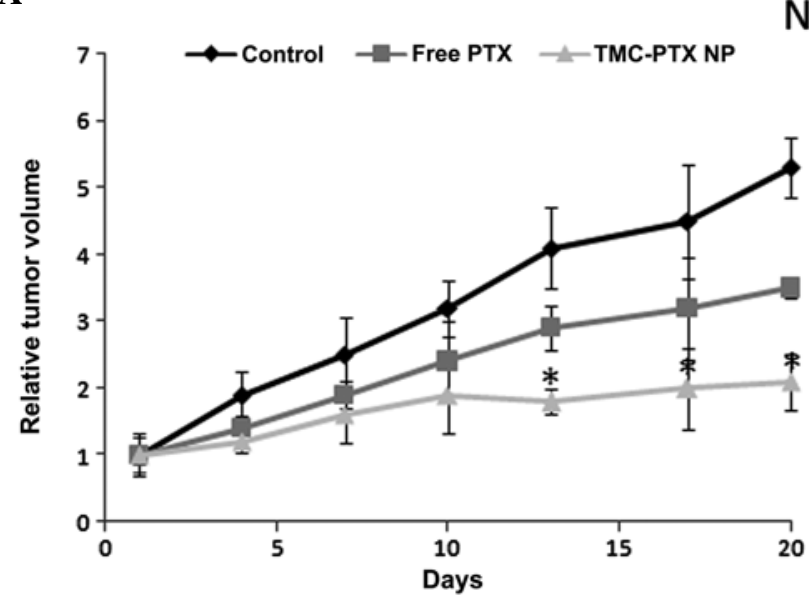

$\mathrm{NCl}-\mathrm{N} 87$

B
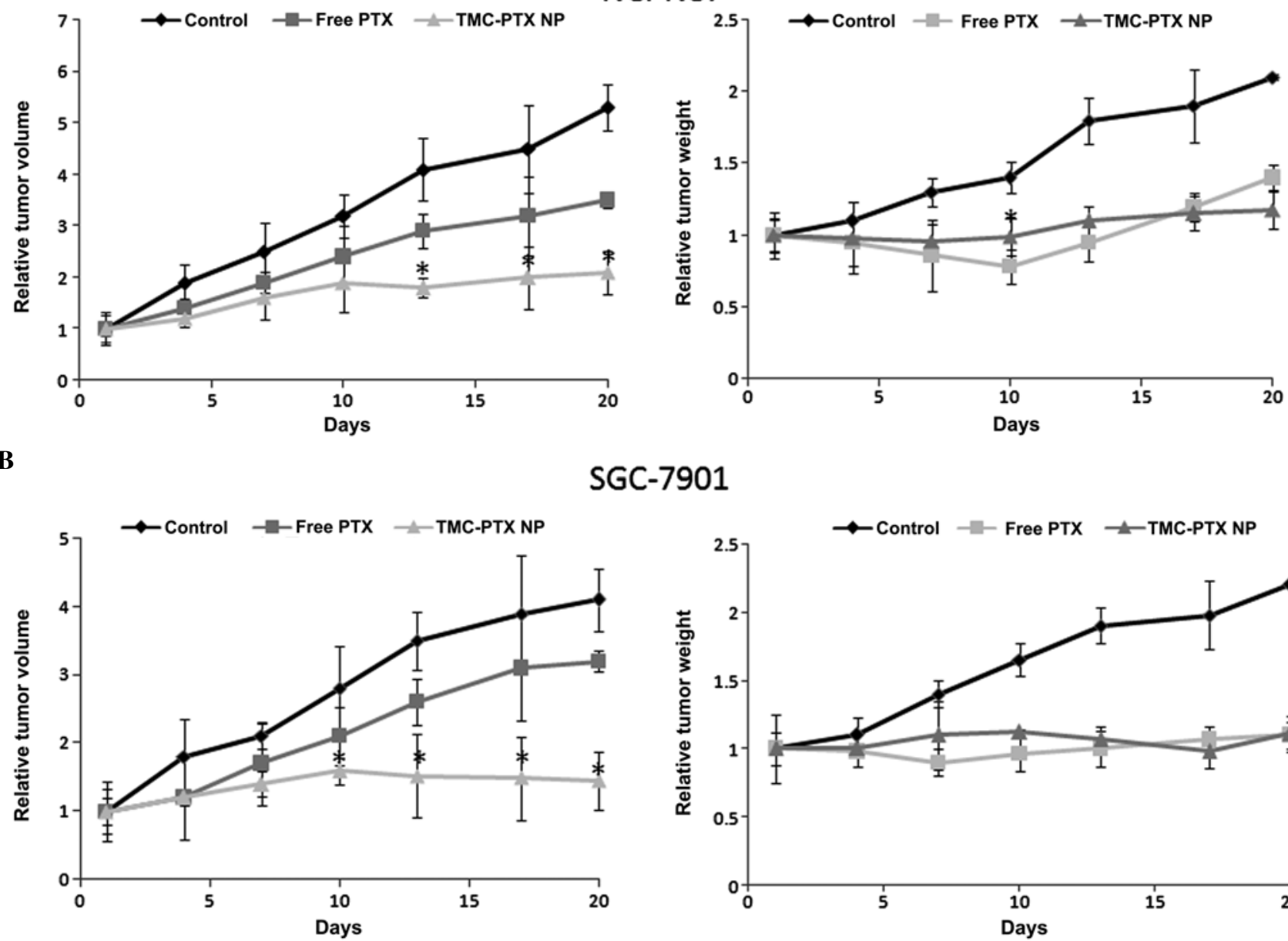

SGC-7901

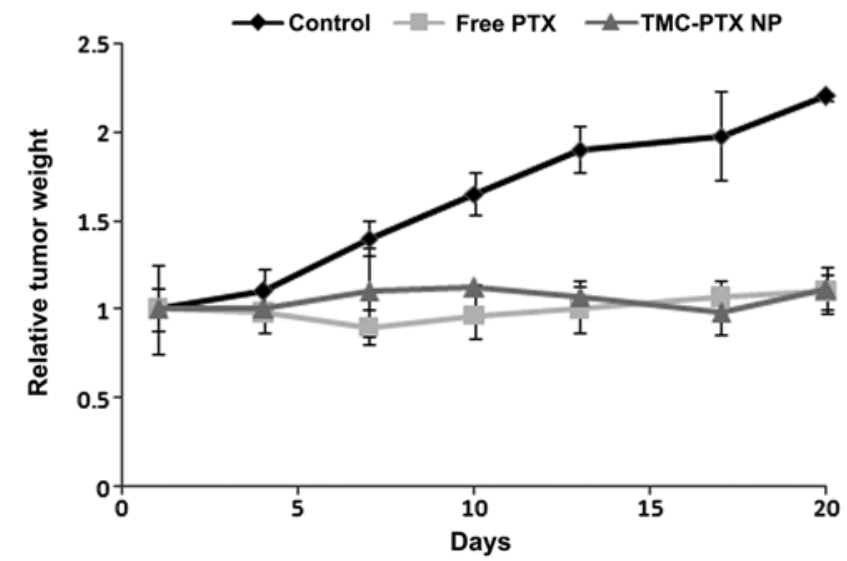

Figure 8. Tumor volume reduction and body weight changes upon subcutaneous injection of free PTX and TMC-PTX NP in (A) NCI-N87 and (B) SGC-7901 gastric cancer cell lines. TMC-PTX, trimethyl chitosan-loaded paclitaxel.

it can be interpreted that free PTX was toxic and mice shed at least $20 \%$ of their weight in the NCI-N87 model indicating a severe toxicity at the present dosage form $(5 \mathrm{mg} / \mathrm{kg})$. In contrast, TMC-PTX was completely safe and at $5 \mathrm{mg} / \mathrm{kg}$ twice a week was well tolerated and the same body weight was maintained without any overt signs of toxicity throughout the study period. In the untreated group, body weight constantly increased, which may have been due to the growth of tumor. When the tumor mass was excised, the mean tumor weight from TMC-PTX group was significantly smaller compared to other groups.

The enhanced antitumor efficacy with appreciable safety profile could be explained on several bases; first, it can be anticipated that NP incorporation of PTX (TMC-PTX) could have greatly improved the blood circulation time resulting in preferable accumulation in tumor tissues via EPR effect (29). Secondly, sustained release pattern of TMC-PTX enabled the slow release of drug which could enter the tumor cells gradually. Thirdly, nanosized particle of $\sim 150 \mathrm{~nm}$ could escape from the reticuloendothelial system (RES) and can easily penetrate the tumor fenestration (30). Therefore, our results clearly reveal the superior antitumor efficacy of TMC-PTX in experimental gastric cancers and appears to be a potent single drug chemotherapeutic agent in clinical settings. These observa- tions support the clinical evaluation of polymer-loaded PTX as a significant microtubule inhibiting agent.

In conclusion, TMC polymer was synthesized and TMC-PTX NP was successfully prepared. The nanosized particles $(\sim 150 \mathrm{~nm})$ were formed with uniform size distribution and spherical dimensions. The TMC NP exhibited a sustained release profile for PTX with $75 \%$ of drug release by $96 \mathrm{~h}$ at physiological $\mathrm{pH}$. Augmented internalization of NP across Caco-2 cell monolayers was confirmed by the HPLC method. The PTX-bound NP showed potent cytotoxic effect in both gastric cancer cells, NCI-N87 and SGC-7901, while the former were more sensitive to PTX than the latter. The cytotoxic effect was further confirmed by apoptosis assay, which clearly revealed the majority of cell populations in early and late apoptosis chamber. Consistently, TMC-PTX showed prominent 60 and $50 \% \mathrm{G} 2 / \mathrm{M}$ phase arrest in NCI-N87 and SGC-7901 cells. Most importantly, it showed 70 and 60\% tumor regression in these cell lines with no obvious signs of any systemic toxicity. The augmented chemotherapeutic effects were due to the preferential accumulation of NPs in the gastric tumor cells via EPR effects. Collectively, the present study highlighted the therapeutic efficiency of TMC-PTX in the treatment of experimental gastric cancer with the possibility of clinical application. 


\section{Acknowledgements}

The authors thank Dr Xian for proofreading the manuscript. The authors also thank the hospital staff members for suggestions and advice during the course of the study.

\section{References}

1. Oh SC: Update of adjuvant chemotherapy for resected gastric cancer. J Gastric Cancer 12: 3-6, 2012.

2. Schwarz RE and Smith DD: Clinical impact of lymphadenectomy extent in resectable gastric cancer of advanced stage. Ann Surg Oncol 14: 317-328, 2007.

3. Jemal A, Bray F, Center MM, Ferlay J, Ward E and Forman D: Global cancer statistics. CA Cancer J Clin 61: 69-90, 2011.

4. Rowinsky EK, Cazenave LA and Donehower RC: Taxol: a novel investigational antimicrotubule agent. J Natl Cancer Inst 82: $1247-1259,1990$.

5. Mancuso A, Oudard S and Sternberg CN: Effective chemotherapy for hormone-refractory prostate cancer (HRPC): present status and perspectives with taxane-based treatments. Crit Rev Oncol Hematol 61: 176-185, 2007.

6. Weiss RB, Donehower RC, Wiernik PH, Ohnuma T, Gralla RJ, Trump DL, et al: Hypersensitivity reactions from taxol. J Clin Oncol 8: 1263-1268, 1990.

7. Akhlaghi SP, Saremi S, Ostad SN, Dinarvand R and Atyabi F: Discriminated effects of thiolated chitosan-coated pMMA paclitaxel-loaded nanoparticles on different normal and cancer cell lines. Nanomedicine 6: 689-697, 2010.

8. Zhang W, Shi Y, Chen Y, Yu S, Hao J, Luo J, Sha X and Fang X: Enhanced antitumor efficacy by paclitaxel-loaded pluronic P123/F127 mixed micelles against non-small cell lung cancer based on passive tumor targeting and modulation of drug resistance. Eur J Pharm Biopharm 75: 341-353, 2010.

9. de Britto D and Assis OBG: A novel method for obtaining a quaternary salt of chitosan. Carbohydr Polym 69: 305-310, 2007.

10. Slütter B and Jiskoot W: Dual role of $\mathrm{CpG}$ as immune modulator and physical crosslinker in ovalbumin loaded $\mathrm{N}$-trimethyl chitosan (TMC) nanoparticles for nasal vaccination. J Control Release 148: 117-121, 2010.

11. Subbiah R, Ramalingam P, Ramasundaram S, et al: N,N,Ntrimethyl chitosan nanoparticles for controlled intranasal delivery of HBV surface antigen. Carbohydr Polym 89: 1289-1297, 2012.

12. Hamman JH, Stander M and Kotze AF: Effect of the degree of quaternisation of $\mathrm{N}$-trimethyl chitosan chloride on absorption enhancement: in vivo evaluation in rat nasal epithelia. Int J Pharm 232: 235-242, 2002.

13. de Britto D, Forato LA and Assis OBG: Determination of the average degree of quaternization of N,N,N-trimethylchitosan by solid state C-13 NMR. Carbohydr Polym 74: 86-91, 2008.

14. de Moura MR, Aouada FA, Avena-Bustillos RJ, McHugh TH, Krochta JM and Mattoso LHC: Improved barrier and mechanical properties of novel hydroxypropyl methylcellulose edible films with chitosan/tripolyphosphate nanoparticles. J Food Eng 92: 448-453, 2009

15. de Britto D, de Moura MR, Aouada FA, Mattoso LHC and Assis OBG: N,N,N-trimethyl chitosan nanoparticles as a vitamin carrier system. Food Hydrocoll 27: 487-493, 2012.
16. Bhumkar DR and Pokharkar VB: Studies on effect of $\mathrm{pH}$ on cross-linking of chitosan with sodium tripolyphosphate: a technical note. AAPS PharmSciTech 7: E50, 2006.

17. Chen F, Zhang ZR and Huang Y: Evaluation and modification of $\mathrm{N}$-trimethyl chitosan chloride nanoparticles as protein carriers. Int J Pharm 336: 166-173, 2007.

18. Gan Q and Wang T: Chitosan nanoparticle as protein delivery carrier - systematic examination of fabrication conditions for efficient loading and release. Colloids Surf B Biointerfaces 59: 24-34, 2007.

19. Qi L, Xu Z, Jiang X, Hu C and Zou X: Preparation and antibacterial activity of chitosan nanoparticles. Carbohydr Res 339: 2693-2700, 2004.

20. Davda J and Labhasetwar V: Characterization of nanoparticle uptake by endothelial cells. Int J Pharm 233: 51-59, 2002.

21. Yu B, Zhang Y, Zheng W, Fan C and Chen T: Positive surface charge enhances selective cellular uptake and anticancer efficacy of selenium nanoparticles. Inorg Chem 51: 8956-8963, 2012.

22. Ganesh T, Yang C, Norris A, et al: Evaluation of the tubulin-bound paclitaxel conformation: synthesis, biology, and SAR studies of C-4 to C-3' bridged paclitaxel analogues. J Med Chem 50: 713-725, 2007.

23. Sharma AK, Zhang L, Li S, Kelly DL, Alakhov VY, Batrakova EV and Kabanov AV: Prevention of MDR development in leukemia cells by micelle-forming polymeric surfactant. J Control Release 131: 220-227, 2008.

24. Guo DD, Moon HS, Arote R, Seo JH, Quan JS, Choi YJ and Cho CS: Enhanced anticancer effect of conjugated linoleic acid by conjugation with Pluronic F127 on MCF-7 breast cancer cells. Cancer Lett 254: 244-254, 2007.

25. Guo DD, Xu CX, Quan JS, Song CK, Jin H, Kim DD, Choi YJ, Cho MH and Cho CS: Synergistic anti-tumor activity of paclitaxel-incorporated conjugated linoleic acid-coupled poloxamer thermosensitive hydrogel in vitro and in vivo. Biomaterials 30: 4777-4785, 2009.

26. Ahmed F, Pakunlu RI, Brannan A, Bates F, Minko T, Discher DE: Biodegradable polymersomes loaded with both paclitaxel and doxorubicin permeate and shrink tumors, inducing apoptosis in proportion to accumulated drug. J Control Release 116: 150-158, 2006.

27. Ng SSW, Tsao MS, Chow S and Hedley DW: Inhibition of phosphatidylinositide 3-kinase enhances gemcitabine-induced apoptosis in human pancreatic cancer cells. Cancer Res 60: 5451-5455, 2000

28. Kim K, Kim JH, Park H, Kim YS, Park K, Nam H, et al: Tumor-homing multifunctional nanoparticles for cancer theragnosis: simultaneous diagnosis, drug delivery, and therapeutic monitoring. J Control Release 146: 219-227, 2010.

29. Zhang L, He Y, Ma G, Song C and Sun H: Paclitaxel-loaded polymeric micelles based on poly( $\varepsilon$-caprolactone)-poly(ethylene glycol)-poly( $\varepsilon$-caprolactone) triblock copolymers: in vitro and in vivo evaluation. Nanomedicine 8: 925-934, 2012.

30. Xiao K, Luo J, Fowler WL, Li Y, Lee JS, Xing L, Cheng RH, Wang L and Lam KS: A self-assembling nanoparticle for paclitaxel delivery in ovarian cancer. Biomaterials 30: 6006-6016, 2009. 